Aus dem anatomischen Institut zU Giessen.

\title{
EXPERIMENTELL ERZEUGTE
}

\section{RÜCKBLLUNGSERSCHENUNGEN AN EIFOLLIKELN}

VON

LACERTA AGILIS.

VON

JOH. AUG. MEYER,

G]ESSEN.

Mit 8 Figuren auf den Tafeln $X X X V I I X X X I X$. 

In einer früheren Veröffentlichung ${ }^{1}$ ) in diesen Heften Bd. XI Heft I habe ich über Zerfallsvorgänge an Ovarialeiern von Lacerta agilis berichtet. Dieselben laufen ab unter Erscheinungen, die eine geraume Zeit in Anspruch nehmen, ehe sie nur erst makroskopisch sichtbar werden, dann aber sich durch Monate hindurch verfolgen lassen. Sie erstreckten sich auf das allmähliche Schwinden des Keimbläschens, dem Lageveränderungen desselben vorhergehen, sodann auf Zerklüftungen des Protoplasma. Letzteres lieferte Bilder, die an Furchungsvorgänge erinnerten und Berührungspunkte mit solchen aufwiesen. Wir konnten aber nachweisen, dass es sich um reine Zerfallsvorgänge handelt, bedingt durch chemisch-physikalische Einflüsse, die mit der Furchung nichts zu thun haben.

Des ferneren haben wir damals Dotterveränderungen beschrieben, die ein weiteres Stadium in der regressiven Metamorphose darstellten. Diese gingen mit einer Resorption des Dotters einher und führten dadurch schliesslich zu einer Verkleinerung des Eies. Für das gleichzeitige Auftreten von Säcken, die sich durch irgendwelche Einflüsse aus mehreren Eieru gebildet hatten, suchten wir Erklärungen zu geben.

Was die mikroskopischen Veränderungen anbelangt, so verfolgten wir in systematischer Untersuchung ebenfalls die Kern-

1) Ủber Zerfallsvorgänge an Ovarialeiern von Lacerta agilis. (Aus dem anatomischen Institut zu Giessen.) 
erscheinungen, Protoplasma- und Dotterzerfallsvorgänge. Hand in Hand damit gehen dann wichtige Veränderungen der Epithelien an den einzelnen Follikelwänden. Diese waren es, die in einer Reihe von Abbildungen des näheren wiedergegeben wurden. Wir konstatierten die Wucherung der Epithelien, das Einwandern von Wanderzellen, das vereinzelte Loslösen der Epithelien von der Wand und ihr Verbleiben in späterer Zeit der Umwandlung.

Schliesslich verglichen wir damals die gewonneuen Resultate mit Beobachtungen von $R$ u g e über Veränderungen am Eifollikel von Amphibien, von v. Brunn und Lau über die von Vogelovarien. Manche Berührungspunkte, aber auch manche Unterschiede fanden sich.

Leider fehlten uns damals die letzten Stadien der Rückbildung. Unsere Beobachtungen erstreckten sich über ein Jahr der regressiven Metamorphose, liessen aber die Frage nach dem endgültigen Schicksal der Eier offen, da die Rückbildungen nach dieser Zeit noch nicht vollständig abgelaufen waren, und die Eidechsen sich nicht länger als etẉa ein Jahr in der Gefangenschaft lebend erhalten liessen.

Da die Aussicht, auf dem bisher betretenen Wege die Endstadien der Umwandlungen zu bekommen, ziemlich gering erschien, so haben wir versucht, in Rückbildung begriffene Ovarialeier auf anderem Wege zu erhalten.

Wir verschafften uns vom Monat April an eine Anzahl weiblicher Eidechsen, in zwei Jahren etwa vierzig Stück. Dieselben wurden isoliert vom Männchen gehalten und eine Befruchtung war auszuschliessen. Auch in der Freiheit vorher hatte eine solche sicherlich noch nicht stattgefunden, weil einmal einige Zeit nach dem Winterschlaf vergeht, ehe zur Befruchtung geschritten wird. Ende April zur Kontrolle getötete Tiere waren sicher unbefruchtet; auch zeigte der Hode aus dieser Zeit keine Spur von Spermatogenese; weil ferner aber auch die kalten und nassen Monate der Frühahre 1901 und 1902 
besonders dazu geeignet erschienen, eine Verzögerung herbeizuführen. Die Tiere wurden gut ernährt und gefüttert, überhaupt den natürlichen Verhältnissen möglichst ähulich gehalten.

Es setzten dann die ersten Veränderungen an den Ovarien mit grossen reifen Eiern Eude Juli, anfangs August ein, wie wir schon früher bemerkten. In dieser Zeit wurden an einer Anzahl von Tieren durch einen kleineren Einschnitt an der seit. lichen Bauchregion die Ovarien, die sofort sichtbar werden, freigelegt. Dabei hatten wir dann Gelegenheit, die früheren $\mathrm{Be}$ obachtungen zu kontrollieren, auf die wir noch zurückzukommen haben. Dann wurden einige der Eier angestochen, so dass ein mehr oder minder grosser Teil des Dotters derselben ablief, und zwar nach aussen und natürlich auch in die Bauchhöhle. Eine Naht schloss sodann die kleine Bauchwunde. Fast alle Tiere überstanden den Eingriff gut.

An einer zweiten Gruppe von Eidechsen, deren Ovarien nur kleine, höchstens $0,6 \mathrm{~cm}$ im Durchschnitt messende Eier enthielten, verfuhren wir ebenso. Diese Follikel zeigten zur Zeit der Operation noch keine Zeichen der Rückbildung im Gegensatz zu den oben erwähnten.

In gewissen Zwischenräumen hinterher und zwar vom ersten Tage nach dem Eingriff bis zum neun und dreissigsten wurden sodann die Eidechsen getötet und ihre Ovarien konserviert.

Falls - wie wir hofften und wie die spätere Untersuchung bestätigt hat - die Reaktion auf den Eingriff in einer Beschleunigung schon vorhandener Rückbildungserscheinungen bei der ersten Gruppe, oder in dem Einsetzen von solchen bei der anderen bestand, musste es auf diesem Wege auch gelingen, nicht nur neues Beobachtungsmaterial an sich, sondern auch die fehlenden Endstadien zu erhalten.

Wir haben, wie schon gesagt, bei Vornahme der Operation an einer ganzen Anzahl von Ovarien Gelegenheit gehabt, die früheren Beobachtungen zu kontrollieren. Wir fanden in solchen 
Fällen das Fehlen der Keimbläschen, die leichten Dotterveränderungen, also Befunde, die die seitherigen nur bestätigen. Auch verschieden grosse Eier fanden sich, wie es natürlich ist, an ein und demselben Ovarium, das stets zeitlich verschiedene Stadien der Reifung enthält. Ging die Grösse der einzelnen Eier über ca. 0,6 cm hinaus, waren jene Umwandlungen sichtbar, sonst nicht. Die Farbe der verschiedenen Eier wechselte ebenfalls, vor allem an den kleinen Formen; wasserhelle fanden sich neben dunkelgelben.

Bei den in verschiedener Zeit nach der Operation getöteten Tjeren war die angestochene Stelle nur in seltenen Fällen durch aufgelagerten Dotter zu. erkennen. Die Form und Lageverhältnisse der operierten Ovarien geben besser wie lange Auseinandersetzungen die beigefügten von Professor Strahl angefertigten Photogramme wieder. Es zeigt Fig. 1 - Ovarium vom 23. Tage nach der Operation - einen Fall, in welchem die bindegewebige Grundlage des Eierstockes besonders ausgiebig zerstört war. Einzelne Eier erschienen ganz vom Eierstock losgelöst und hingen nur noch durch einen bindegewebigen Stiel untereinander und mit dem Ovarialrest zusammen. Was vor allem auffällt, ist die äusserst reichliche Gefässwucherung an dem einen Ei, während die rauhe Oberfläche eines zweiten durch aufgelagerten Dotter bedingt war. Im übrigen zeigen gerade die beiden losgelösten Eier einen geringeren Grad der Umwandlung -- besonders in den Grössenverhältnissen - gegenüber der Mehrzahl der gleichalterigen Follikel.

Fig. 2 giebt ein Stück der hinteren Leibeswand einer Eidechse wieder, welche einunddreissig Tage nach der Operation getötet wurde. An derselben hingen ein normales Ovarium (a) und das operierte (b).

An letzterem sitzen oben und unten (c) die Reste von angestochenen Eiern als kleine unregelmässig gestaltete Vorsprünge zwischen wenig entwickelten normalen Eiern. 
Experimentell erzengte Rückbildungserscheinungen an Fifollikeln etc. 583

In Gegensatz zu Fig. 1 zeigt diese Abbildung bei $c$ sehr stark verkleinerte und in der Rückbildung weit vorgeschrittene Eier; der Vergleich mit dem gesunden nicht operierten Ovarium ist ebenfalls besonders instruktiv.

Das folgende Photogramm (Fig. 3) zeigt ein noch älteres Stadium. Hier waren beide Ovarien vor 23 resp. 35 Tagen operiert. Zusammengeklappte rauhe Stellen mit Vorsprüngen und Einbuchtungen $(a-d)$ zeigen die Stellen an, wo vordem die grossen Eier, die damals angestochen wurden, sassen. Gut erhaltene kleine Eier sind sichtbar geblieben, da diese nicht verletzt waren.

Als regelmässiger Befund kann die Thatsache verzeichnet werden, dass der Grad der Form- oder Gestaltsveränderung in erster Linie abhängig ist von der Menge des ausgetretenen Dotters. So finden wir denn länglich ovale Formen neben stärker abgeplatteten, an denen sich die $\mathrm{W}$ ände einfach aneinauder gelagert haben. Ferner sind Einsenkungen der Eiwände in das Lumen an fast allen Eiern zu verfolgen und zwar ebenfalls in verschiedenem Grade je nach der Ausdehnung. Im übrigen verweisen wir auf Fig. 2 und 3, an denen einzelne dieser Veränderungen ohne weiteres $\mathrm{zu}$ sehen sind.

War bei der Operation viel Dotter in die Bauchhöhle ge. langt und erfolgt die Tötung in nicht zu langer Zeit, etwa bis zu 20 Tagen hinterher, dann fanden sich auf den Eiern reichlich Auflagerungen von verändertem Dotter, oft fadenförmig angeorduet, oft in Flocken zusammen liegend; vor allem sah man dies in den Buchten zwischen den einzelnen Eiern und im Mesovarium. Wir haben hierauf bei Beschreibung der mikroskopischen Befunde noch zurückzukommen.

Die Farbe der operierten Ovarien der getöteten Tiere weicht in einigen Fällen von der bei der Operation verzeichneten ab, hat sich also in der Zeit von der Operation bis zur Tötung geändert. Es fanden sich wiederholt grünlich-bräunliche Eier, solche, die ganz, und solche, die nur an den der ventralen 
Leibeswand gegenüberliegenden Kuppen verfärbt waren. In einem Falle war das Ei grün und die Kuppe braun. Ein Teil dieser eigentümlichen Verfärbungen ist auf Veränderung extravasierten Blutes zurückzuführen.

Makroskopische Unterschiede zwischen Eiern, die, bereits in Rückbildung begriffen, operiert wurden und den kleinen, der anderen Gruppe angehörigen, die in gesundem Zustande zur Operation kamen, fanden sich nicht.

Wir wenden uns nunmehr zu den mikroskopischen Veränderungen, wie sie zeitlich verschieden sich verfolgen lassen, um ein Bild von den Folgen des Eingriffes ebenfalls an beiden Gruppen von Ovarien zu bekommen.

Zunächst lassen sich da die makroskopisch festgestellten Formund Gestaltsveränderungen je nach der Menge des noch vorhandenen Dotters um vieles besser übersehen, die Schnitte bestätigen aber das bereits Gesagte.

Im übrigen kommen, da das Ei durch den Eingriff zerstört wird, für die mikroskopischen Bilder wesentlich die Umwandlung der Follikel in Frage, neben diesen allerdings auch die Veränderungen der Eireste.

In den ersten Tagen nach der Operation finden wir an unseren Schnitten reichlich freie Blutkörperchen, die einfach ausgetreten sind, als Folge des Eingriffes. Ferner sehen wir, dass der Dotter sich sowohl an der Aussenseite des Eies festgesetzt hat, als auch überhaupt das Mesovarium mehr oder weniger erfüllt. Die Follikelepithelien, die bei den früher beschriebenen Zerfallsvorgängen erst frühestens Ende September sich auf dem Höhepunkte der Ausbildung befanden, erreichen bei der jetzigen Anordnung der Versuche ihre grösste Form schon erheblich früher. Etwa im Verlaufe von zehn Tagen nach dem Eingriff finden wir alle Formen, die den früheren Abbildungen zu Grunde lagen, vertreten; somit Wucherung, Schichtenbildung, Dotterauf nahme und schliesslich Kernzerfall. 
Es muss indessen hier erwähnt werden, dass nicht an allen Eiern sich die Epithelien regen und diese Veränderungen aufweisen; in einigen Fällen reagiert das Epithel überhaupt nicht. Über die Verschiedenheit der Wirkung kann man nur Vermutungen haben; so könnte die mehr oder weniger starke Läsion der Wände, oder auch eine leichte Infektion das Wechseln der Bilder bedingen.

An das Stadium des nukleären Kernzerfalles, das damals als erstes beschrieben ist, können wir nunmehr, wegen der Ähnlichkeit der Erscheinungen, anschliessen. Die grossen zerfallenden Epithelien finden wir hier bereits nach zehn Tagen und dann können wir an älteren Eiern sie verfolgen bis etwa zum Schlusse der fünften Woche. Neben den zerfallenden sehen wir aber auch solche, die auf einem früheren Stadium der Wucherung einfach in ihrer Entwickelung stehen bleiben. Auch diese erhalten sich bis an das Ende der fünften Woche, wo sie in nur wenigen Exemplaren noch zu sehen sind. Ferner erhalten sich kleinere Komplexe von normalen Epithelien von Anfang an bis ebenfalls in diese Zeit.

Für das Vorkommen dieser verschiedenen Formen nebeneinander können wir eine Erklärung nicht geben; vielleicht ist daran zu denken, dass, noch ehe der Kern in den grössten Formen degeneriert, sich durch Rückbildung wieder normalen Zuständen näher kommende Epithelien bilden können oder aber daran, dass den Zellen verschiedene Leistungen zufielen, die sich durch die Verschiedenheit ihrer Form dokumentieren.

Wenn früher gesagt wurde, dass die Hauptthätigkeit und Aufgabe der Epithelien, sowohl der losgelösten intravitellinen als auch der in der Wandung befindlichen in der Aufnahme und Verarbeitung von Dotterkörnchen beruht, so bin ich nach obigen Befunden, die aus dem operativen Material gewonnen, geneigt, diese Meinung etwas einzuschränken. Es stimmt ein- 
mal die Menge des noch zu verarbeitenden Dotters nicht immer mit der Menge der vorhandenen Epithelien; in dem Falle, wo viel Dotter vorhanden, sind oft wenig mit seiner Resorption beschäftigte Follikelepithelien zu sehen, und umgekehrt, nachdem aller Dotter längst verschwunden, sind noch Zellen vorhanden.

Ausserdem treten, ehe noch der Dotter vollständig aufgelöst ist, neue Faktoren in Erscheinung, die nach unserer Meinung nunmehr jene Zellen in ihrer Aufgabe ablösen, wir meinen das massenhafte bis dahin bei Reptilien nicht beobachtete Einwandern von Gefässen, auf das wir gleich zurückkommen werden. Somit leiten wohl, alle Momente zusammengefasst, bei unseren Präparaten wenigstens die Epithelien nur den Zerfall ein, gehen teilweise $\mathrm{zu}$ Grunde, dienen teilweise $\mathrm{zu}$ anderen $Z$ wecken, so dass die Blutgefässe ihre Rolle aufnehmen und fortsetzen müssen. - Letztere treten also in dem Stadium des nukleären Zerfalles in Erscheinung. Makroskopisch waren sie nicht an allen Eiern zu sehen, um so besser zeigt das Mikroskop ihr Verhalten. Sie rücken von der Seite, wo das Ei sich noch in Verbindung mit der Wand des Eierstockes befindet, an das Ei heran.

Dann gelangen sie in die versehiedenen Schichten der Wand, anfänglich mehr in die bindegewebigen, wo sie mehrere Lagen übereinander bilden können, die dann durch ein ganz feines Septum getrennt siud. Man sieht hier an den zusammengeklappten Wänden ein prallgefülltes Gefäss am auderen. Zwischen den Epithelien sind sie anfangs in der Minderzahl und nur in kleinen Durchschnitten zu sehen. Stellenweise sieht man gar keine Wandungen, sondern nur eine feine dunkle Linie als Abgrenzung gegenüber dem Protoplasmaleib der Epithelien. Dass es sich auch in solchen Fällen um Blutgefässe handelt, kann aus dem Inhalt geschlossen werden, der sich ohne jeden Zweifel als Blutkörperchen erweist. Diese Verhältnisse gehen noch weiter. Fiel das erste Auftreten in das Ende der zweiten und in die ganze dritte Woche, so zeigen Präparate aus dem An 
Experimentell erzengte Rückbildungserscheinungen an Eifollikeln etc. 587

fange der vierten Gefässsprossen, die blind endigen, bis zwischen die intravitellinen Epithelien hinein, also ein weiteres Stadium in der Umwandlung.

In der Umgebung solcher Sprossen sieht man massenhafte rote und weisse Blutkörperchen, die sich von den Epithelien wohl unterscheiden lassen, eine Thatsache, die auch schon Ruge bei seinen Untersuchungen konstatieren konnte. Sie durchsetzen in der Folge mit den ebenfalls seit dem Eingriff dort liegenden weissen Blutkörperchen, an denen sich noch kein Zerfall nachweisen lässt, den Dotter in allen möglichen Entwickelungsphasen und finden sich schliesslich über den ganzen Inhalt des ursprünglichen Eies mehr oder weniger zerstreut vor. Da in diesem Stadium die Lebensfähigkeit der Follikelepithelien, soweit sie sich im Kernzerfall befinden, sicherlich aufgehoben ist, finden sie keine Schwierigkeiten, wie bei dem im ganzen Umfange erhaltenen Epithel, und so durchwachsen sie von den toten in Auflösung begriffenen Teilen aus das ganze $\mathrm{Ei}$, und „wir sind nicht überrascht, sie im ganzen Eiinhalt zu finden".

Hier inmitten des Dotters sind an den Gefässen auch die Endothelien der Wand zu erkennen. Waren diese Verhältnisse schon erkennbar an Eiern, deren Gestalt- und Formveränderungen nur geringe Abweichungen von der Norm boten, so treten sie in noch höherem Masse an denen hervor, welche die schon einmal erwähnten Verhältnisse in stärkerer Ausbildung zeigen. Bei diesen, den abgeplatteten und mit Einbuchtungen versehenen Eiern treten Teile der Wandung in das Lumen hinein, um dort noch mehr ihr Zerstörungswerk fortsetzen zu können. Dann sieht man fast ganz abgeschnürte Teile und von ihnen ausgehend wiederum die Gefässe, in deren Umgebung sich in Masse die Blutkörperchen finden. Zwischendurch sind dann auch die ganz losgelösten Follikelepithelien in den verschiedenen Stadien zu sehen. - Diese Vorgänge füllen die vierte Woche nach der Operation aus. An unseren Schnitten, von Präparaten aus der 
vierten Woche nach der Operation gewonnen, ist in Bezug auf die Umwandlung ein weiterer Fortschritt zu vermerken. Bei diesen ist der ganze Inhalt gleichmässig verändert. Gefässe und Blutkörperchen füllen ibn aus, so dass letztere neben den schon erwähnten Epithelien in ihrer verschiedenen Gestalt an der Peripherie, an der Grenze von Peripherie und Dotter und in diesem selbst sich finden. Die feineren Verhältnisse der weissen Blutkörperchen ändern sich in dieser Zeit ebenfalls. Alle Um. wandlungen, welche für Epithelien beschrieben sind, treten auch an den Wanderzellen auf, so dass auch diese, nachdem sie Dottermassen zur endgültigen Verarbeitung vorbereitet und resorbiert haben, dem Untergange geweiht sind und der Auflösung verfallen.

$\mathrm{Zu}$ denselben Resultaten bei der Rückbildung von Amphibien-Eiern war Ruge in seiner bereits mehrfach erwähnten Arbeit schon früher gekommen. In der ersten von mir veröffentlichten Abhandlung hatte ich aus Mangel an Material diese letzten Vorgänge nicht nacharbeiten können.

Jetzt, wo ich an den operierten Eierstöcken dasselbe finde, glaube ich dem Autor gerecht $\mathrm{zu}$ werden, wenn ich von neuem auf dessen mit zahlreichen Abbildungen ausgestattete Arbeit über Amphibien hinweise; besonders die Abbildungen sind so klar und die verschiedenen Entwickelungsphasen über den Werdegang der Leukocyten so überzeugend nebeneinander gestellt, dass ich nur nochmals auf die Darstellung Ruges verweisen kann.

Im Vorhergehenden versuchten wir Follikelepithel und Leukocyten in ihrer Thätigkeit der Dotterumwandlung und der Resorption zu verfolgen. Es erübrigt sich für uns, des näheren auf den Dotter selbst einzugehen.

Ursprünglich besteht dieser aus den bekannten Kugeln. Diese werden in früher Zeit nach der Operation umgewandelt und zum Teil gelöst; dann sieht man breite helle Strassen durch 
die Dottermasse ziehen, deren Seiten mit Blutkörperchen besetzt sind. Da, wo diese Strassen an Epithelien stossen, zeigen sie Lacunen, die, wie an anderem Orte erwähnt, durch Thätigkeit letzterer entstanden anzunehmen sind. Mit der Zeit, in der zweiten und dritten Woche, werden die homogenen Strassen breiter und breiter, d. h. es werden wohl immer mehr Dotterkugeln aufgelöst und die aufgelösten Teile senken sich, wie wir mehrfach zu beobachten Gelegenheit hatten, in die abhängigen Partien der Eier, so dass sie dem Pole, der an den bindegewebigen Aufhängeapparat stösst, genau gegenüber zu liegen kommen. Aber auch dann ändert sich das Aussehen noch; aus dem ursprünglich wohl flüssigen, durch die Wirkung der Agentien homogen erscheinenden Partien werden weiterhin fein faserige, helle Teile; mit starker Vergrösserung erscheinen diese aus feinsten körnigen Partikelchen bestehend. Um diese Zeit ist die Einwanderung der Epithelien und der Leukocyten bereits beendet. Zahlreich liegen sie in den Dotterteilen; während aber die Zellen an den Punkten, wo der Dotter noch die homogene Beschaffenheit zeigt, in voller Thätigkeit sind, sind sie an den anderen Stellen in Rückbildung begriffen. Es ist dieser Umstand uns ein Beweis dafür, dass mit dem Auftreten der Zerfallserscheinungen an diesen Zellformen der Dotter in seiner Rückbildung den letzten Grad erreicht hat; die Dauer der Umwandlung und der Resorption des Dotters an den grossen operierten Eiern dürfte somit wohl höchstens eine Woche betragen; nach dieser Zeit sind nur noch ganz geringe Reste von Eiweissgerinnsel wahrnehmbar.

War bei der Operation Dotter in die freie Bauchhöhle, an die Aussenseite der Eier und des Mesovarium gelangt, was ja in den meisten Fällen nicht zu vermeiden war, so fanden wir an diesen Stellen ebenfalls die gleichen Stadien des Dotterzerfalles, wie wir sie der Reihe nach beschrieben haben. Die Körnchen wurden auch von dem darmumschliessenden Perito- 
nealepithel aufgenommen, das sich in hohem Masse an der Resorption beteiligte. Es zeigen Querschnitte durch den Darm ein äusseres hohes Epithel, dessen Protoplasmaleib sich mit Körnchen von obiger Beschaffenheit gefüllt hat.

Unter solchen Voraussetzungen beteiligte sich ferner an der Resorption auch die den Eifollikel bedeckende, an gesunden Eieru ganz glatte äussere Schicht. Auch deren Zellen sind hoch geworden und gefüllt mit dem Gerinnsel. Nicht genug damit. Ganze Komplexe dieser Epithelien lösen sich aus ihrem Verbande und finden sich auf den Durchschnitten ziemlich unregelmässig zerstreut überall da, wo Dotter das Mesovarium erfüllt. Sie sind in denselben Stadien zu verfolgen wie das Follikelepithel; dass auch Wanderzellen gemeinsam mit ihnen sich finden, bedarf wohl keiner besonderen Auseinandersetzung. Wenn man nun auch diese Entstehungsweise des äusseren veränderten Epithels annimmt, muss man sich gleichzeitig doch auch fragen, ob nicht von den inneren Epithelien durch irgend welche Insulte diese Zellen nach aussen gelangt sein können. Ich habe aber trotz vielfacher Durchmusterung meiner Präparate denselben keine Anhaltspunkte für einen derartigen Wanderungsvorgang entnehmen können.

Weiter mögen nun die letzten Beobachtungen der Eiumwandlungen hier mitgeteilt sein, die sich auf die fünfte Woche und noch später erstrecken.

Die Eier waren nach den geschilderten Vorgängen, auch wenn sie anfänglich noch eine bedeutende Grösse hatten, kleiner und kleiner geworden. Ihre Wände berühren sich teilweise und stellenweise liegen sie sogar in ganzer Länge aneinander. Solche Bilder finden sich in Präparaten aus dem Ende der vierten und dem Anfang der fünften Woche in Hülle und Fülle. Neu an ihnen ist die augenfällige Erscheinung, dass die Gefässe an Zahl und Ausdehnung noch zugenommen haben, und das Ei, dessen Lumen auf ein Minimum reduziert erscheint, überallhin 
Experimentell erzeugte Rückbildungserscheinungen an Fifollikeln etc. 591

durchziehen. Der bindegewebige Anteil der Follikelwand ist um diese Zeit um das dreifache gewachsen und der epitheliale wohl noch um mehr zurückgegangen. Zeigten die Gefässe anfangs nur gering ausgebildete Wandungen, so ist dieses jetzt anders geworden. Überall sind sie wohl ausgebildet, und selbst die mehr dem Lumen zu gelegenen zeigen an Stelle der fein konturierten Linie oder der Endothelkerne ebenfalls Wandungen, in denen sich verschiedene Zelllagen übereinander gelagert haben. Mit der Ausbildung der Gefässe geht natürlich ein weiteres Verschwinden der Epithelien und der Leukocyten und selbstverständlich auch ihrer Zerfallsprodukte Hand in Hand. In Präparaten aus der vierten Woche konnte man noch von einem epithelialen Wandanteil reden. Jetzt im Verlauf der nächsten Woche sind nur noch einzelne Zellen vorhanden, die hie und dort zerstreut in dem winzigen Lumen sich finden. An Stelle der Epithelien ist der mächtig gewucherte bindegewebige Teil getreten. Anfangs von der Seite, wo das einzelne Ei im $\mathrm{Zu}$ sammenhang mit dem Aufhängeapparat ist, am stärksten wuchernd, finden sich schliesslich auch von den anderen Seiten die Bindegewebssprossen, die von den Gefässen ihren Ausgang nehmen, und schliesslich tritt an die Stelle des Eies nur noch eine kernreiche Masse, die nach dem Innern, dem ursprünglichen Lumen $\mathrm{zu}$, weniger dicht und kernreich ist. Dieses Verhalten der Kerne giebt allein noch Kunde, dass an dieser Stelle ein Ei gesessen hat. Im grossen und ganzen sind diese letzten Stadien alle gleich; überall treten dem Beschauer dieselben Bilder entgegen.

Auch die ins Mesovarium gelangten Dottermassen, die ebenfalls von äusseren Epithelien und Wanderzellen durchsetzt wurden, sind in dieser späteren Zeit ähnlich wie die Eier von Gefässen durchzogen und organisiert; sie bieten dann nur das eine Besondere, dass alle Partien gleichmässig kernreich und ungleichmässig in der Form erscheinen; aus ersterem Verhalten sind sie den Eiresten gegenüber zu erkennen. 
An einigen wenigen Präparaten aus dieser letzten und spätesten Periode der regressiven Metamorphose finden wir Verwachsungen bindegewebiger Natur mit benachbarten Organen z. B. mit Leber und Fettkörper. Ursprünglich waren dieses wohl nur Verklebungen, die sich dann in der Folge zu fester Vereinigung bindegewebiger Art entwickelten, was übrigens nach den geschilderten Vorgängen nicht wunderlich ist.

Der durch den Eingriff geschaffene Reiz erstreckt sich noch über den Bereich des Ovariums hinaus. Wir sehen nämlich an den Präparaten der letzten Stadien - Präparate, die Ovarium und Leiberwand in Zusammenhang geschnitten zeigen - dass die Gefässe von weither aus der Rückenmuskulatur kommen. Sie durchbrechen auf ihrem Wege zum Ovarium die die Leibeshöhle auskleidende Pigmentschicht; dadurch werden Lücken geschaffen, die den Weg abgeben für die Gefässe und auch für die von ihnen abgehenden Bindegewebssprossen. Insgesamt wird dadurch der Aufhängeapparat des Ovariums, sofern von einem solchen überhaupt noch gesprochen werden kann, erheb. lich verbreitert und verdickt.

Die bisher geschilderten Rückbildungsvorgänge fanden sich, wie anfangs erwähnt, an solchen Eierstöcken, die bereits im Anfangsstadium der Metamorphose befindlich operiert wurden. Demgegenüber würden uns nunmehr die Erscheinungen beschäftigen, die an den in gesundem Zustand operierten noch in Reifung befindlichen Eiern vor sich gehen. Im grossen und ganzen kann man sagen, dass die Befunde in den verschiedenen alten Stadien durchaus die gleichen sind. Weil aber die reifenden, noch kleinen, vielfach auch der Dotterkugeln entbehrenden Eier schon von Natur aus reichlicher mit Blutgefässen versehen sind, wie die reifen, auf der Höhe der Entwickelung stehenden Formen, so ist es von vornherein klar, dass die Umwandlung in kürzerer Zeit nach der Operation be- 
endet sein muss. Dafür sorgen eben die reichlicher vorhandenen Blutgefässe. Es kommt hinzu, dass vor allem die Dottermenge, die von dem vor der Operation in mehreren Schichten vorhandenen Epithel zu vernichten ist, in der überwiegenden Mehrzahl dieser kleinen Eier gering und noch nicht zu jenen Schollen und Kugeln geworden ist, wie wir es an den grossen Formen finden.

Was die Zeit selbst anbelangt, so genügen für diese Art von Eiern knapp die Hälfte der Tage, die von den grossen Formen benötigt wurden. Während wir hier erst im Verlauf der fünften Woche und noch später die letzten Stadien zu Gesicht bekamen, finden wir die ersteren nach etwa vierzehn Tagen vollständig degeneriert; in dieser kurzen Zeit war es uns nicht möglich, die Anfangsstadien z. B. von der Keimbläschenrückbildung zu beobachten, weil wir eben mit einer solchen Verkürzung der Regressionszeit nicht gerechnet hatten. Immerhin würden bei der grossen Ähnlichkeit oder Übereinstimmung der späteren und letzten Stadien auch diese früheren wohl die gleichen gewesen sein, wie sie an den grossen Formen sich fanden.

Dass übrigens neben unseren operierten Eiern auch andere kleinere vorkommen, welche spontan in Rückbildung begriffen sind, wollen wir als möglich, schliesslich auch als wahrscheinlich gelten lassen. Dass solche überhaupt im Reptilien-Ovarium vorkommen, hat bereits vor einem Jahrzehnt Arnold (Beiträge zur Kenntnis des Reptilien-Ovariums. Diss. Erlangen 1892) allerdings wohl mehr als gelegentlichen Befund beschrieben. Auch das ganz neuerdings von Wetzel (Arch. f. Anat. u. Phys. Phys. Abt. 1902 Suppl.) beschriebene Auftreten von Kernen der Granulosazellen in Ovarialeiern von Pelias berus dürfte wohl im Sinne einer Degenerationserscheinung aufzufassen sein.

Einer kurzen Erwähnung bedarf auch die Frage des Vorkommens von Pigment. An vielen Präparaten aus der späteren Zeit, aber nicht an allen, beobachtet man inmitten des ursprünglichen Lumens, der späteren kernärmeren Partie, Reste von frei- 
liegendem Pigment. Die Farbe derselben ist gelblich und an dem Aussehen sofort als Überbleibsel von untergegangenen roten Blutkörperchen erkenntlich.

In Epithelzellen liegendes körniges Pigment, wie es Ruge für Amphibien beschrieben, fand sich dagegen nur in einem einzigen Falle und auch da noch sehr spärlich vor. Dieser Fall bot makroskopisch schon die merkwürdigsten Farbenunterschiede. Wenn andere das geringe Vorkommen von Pigment darauf zurückzuführen geneigt sind, dass in späteren Stadien überhaupt eine starke Abnahme des Pigments zu konstatieren sei, so stimmen wir dem nicht bei; in der schon erwähnten Arbeit, welche die frühen Stadien behandelte, konnten wir das Pigment ebensowenig wie jetzt an unserem operativen Material finden, so oft wir auch unsere Serien daraufhin durchmusterten. Einstweilen ist es uns unmöglich, über die Unterschiede in betreff des Vorkommens von Pigment bei den verschiedenen Wirbeltierklassen befriedigenden Aufschluss zu geben.

Wir haben bis dahin die bei der Rückbildung der Eier ablaufenden Vorgänge möglichșt im Zusammenhange dargestellt.

Im nachfolgenden sollen für einzelne der Hauptphasen der Rückbildung Belege durch Abbildungen gegeben werden, die nach Schnittpräparaten der entsprechenden Ovarien hergestellt sind.

Die Figuren schliessen im ganzen an die in meiner früheren Arbeit gegebenen an, sie sind auf Zeichnungen bei geringen Vergrösserungen beschränkt.

Es zeigt Abbildung 4 einen Follikeldurchschnitt von einem Ovarium, welches ebenso wie das gleich zu besprechende Bild 5, zweiundzwanzig Tage nach der Operation gewonnen wurde. Sie schliesst sich ohne weiteres zeitlich an das Bild 10 der erwähnten Abhandlung an. Der bindegewebige Anteil der Follikelwand ist mässig verbreitert, in ihm liegen aber bereits reichlich Ge- 
fässe mit Blut gefüllt. Die Epithelien kleiden als sehr grosse Zellkörper das Lumen noch ringsum aus. Viele sind wohl erhalten, andere im Zerfall begriffen, von dem sich verschiedene Phasen finden. Wir sahen kleine und mittelgrosse, schon wieder zurückgebildete Zellen, letztere vor allem in den beiden Eispitzen oben und unten. Der Inhalt besteht aus feinem Eiweissgerinnsel in geringer Menge.

Die folgende Abbildung 5 giebt einen Durchschnitt durch ein anderes $\mathrm{Ei}$, aber von demselben Ovarium wieder. Hier ist die Follikelwand bereits breiter geworden, so dass die Epithelien stellenweise überwachsen und dadurch in den bindegewebigen Wandanteil verlagert erscheinen. Im übrigen haben die Epithelien sich weiter umgewandelt. Wir finden sie nicht mehr das ganze Lumen auskleidend, sondern nur noch an wenigen Stellen bilden sie einen Überzug (Ep.), der sehr unregelmässig aus den verschiedenen Zerfallsstadien derselben sich zusammensetzt. Im Lumen sieht man reichlich Blutkörperchen, die zum Teil nach Beendigung ihrer Aufgabe ebenfalls in Zerfall geraten. Sonst ist vom Dotter nichts mehr übrig geblieben, als die schon beschriebene feinkörnige Masse.

Ein weiteres Stadium zeigt sodann Abbildung 6. Das Ovarium, von dem es stammt, ist 31 Tage nach der Operation in Behandlung genommen. Die Follikelwand ist breiter geworden. Deutlich sieht man das ältere, dichtere, mebr peripher gelegene Bindegewebe sich von dem jüngeren mehr central gelegenen scheiden. Nur noch wenige Epithelien liegen im Bindegewebe zerstreut. An ihre Stelle sind die immer zahlreicher gewordenen Gefässendothelien getreten, die mit den Leukocyten die Hauptmasse ausmachen, die das ehemalige Lumen ausfüllt. In letzterem ist der letzte Rest des Dotters als feine schmale Platte zu sehen, an deren Rändern noch Wanderzellen liegen, die nunmehr sämtlich degeneriert sind. 
Das in der regressiven Metamorphose eine wiederum ältere Stufe bildende Präparat, dem Bild 7 entnommen ist, zeigt überhaupt nur noch den bindegewebigen Anteil der Wand ohne Epithelien. Weil es ein grosses Ei war, ist ein Lumen allerdings noch zu erkennen; dieses zeigt feinste Bindegewebssprossen, die ursprünglich von den Gefässen ihren Ausgang genommen, von deren grosser Zahl man sich überzeugen kann. Sehr schön tritt in Erscheinung die auf einer früheren Stufe der Umwandlung erfolgte Zusammenfaltung ganzer Wandstrecken, die zu einer erheblichen Einengung des Lumens getührt haben. Die kernreicheren innen gelegenen Teile lassen sich stellenweise ebenfalls an den äusseren unterscheiden.

Als letztes Bild fügen wir 8 hinzu. Dasselbe ist vom Eierstock eines Tieres, das achtunddreissig Tage nach der Operation getötet wurde. Es giebt das Endstadium der Umwandlung wieder. Das ganze Mesovarium ist mitdargestellt, a ist die Kuppe eines gesunden Eies, b und c sind Reste von zurückgebildeten. Dieselben bestehen aus gleichmässig angeordnetem Bindegewebe mit wenig Kernen, hie und da von Gefässen durchzogen. Von Epithelien, von einem Lumen ist nichts mehr zu sehen. Sofern die Eireste nicht, wie c, isoliert sind und dann ohne weiteres als solche gedeutet werden müssen, grenzt, wie in b, eine kernreichere Partie des gefässreichen Mesovariums sie deutlich ab.

Gerade diese letzten Stadien der Metamorphose, von denen wir die Abbildungen entnahmen, sind es, die aufs neue den Vergleich mit dem von Ruge und von Brunn Beschriebenen nahelegen. Sie zeigen uns, dass in den Endstadien mehr und mehr Ähnlichkeiten sich finden, dass Verschiedenheiten bei der Rückbildung sich nur in nebensächlichen Momenten aufrecht erhalten lassen. Des näheren haben wir in unserer Ausführung an manchen Stellen hierauf hinweisen können. - 
Experimentell orzeugte Rückbildungserscheinungen an Eifollikeln etc. 597

Wie gross die Übereinstimmung in Ablauf der Rückbildungsvorgänge bei den verschiedenen Muskeltierklassen sein kann, lehrt auch ein Vergleich mit den soeben erschienenen Untersuchungen von Bühler (Rückbildung der Eifollikel bei Wirbeltieren. I. Fische. Morph. Jahrbuch Bd. XXX. 3. 1902.) Bühler hat sehr eingehend und sorgfältig Rückbildungserscheinungen bei Petromyzon und Coregonus untersucht. Die Zusammenfassung, die er (l. c p. 448) über seine Untersuchungs-Ergebnisse liefert, liesse sich in mancher Beziehung fast wörtlich auf die von 'uns beschriebenen experimentell gewonnenen Bilder übertragen. Auf die Arbeit von Bühler kann ich auch in betreff der Litteraturzusammenstellung verweisen.

Es dürfte im vorstehenden wohl gelungen sein, der früher beschriebenen langen Kette von Rückbildungserscheinungen an Ovarialeiern von Lacerta die letzten noch fehlenden Glieder einzufügen.

Allerdings müssen wir uns bewusst bleiben, dass das Material auf einem von dem früher benutzten abweichenden Weg gewonnen ist unter Zuhilfenahme einer Operation, die immerhin andere Bedingungen schafft; überstanden auch die Tiere den Eingriff gut, so hatten wir es doch nicht in der Hand, im einzelnen die Verletzungen in ihrer Schwere bestimmen zu können. Infolgedessen bedurfte es auch einiger Vorsicht und Auswahl, um aus der Menge der Erscheinungen diejenigen Bilder zusammenzustellen, die mit Sicherheit in Einklang zu bringen waren mit den bisherigen Befunden.

Die thatsächlichen Ergebnisse würden, un kurz zu rekapitulieren, sein: Die auf experimentellem Wege erhaltenen Rückbildungserscheinungen an Ovarialeiern von Lacerta agilis mögen diese Eier nun bereits im Zerfallsanfang befindlich oder im gesunden Zustand operiert werden - sind recht wenig verschieden 
598 J. A. MEYER, Experimentell erzeugte Rückbildungserscheinungen etc.

von denen, welche offenbar physiologisch bei Reptilien ebenso wie bei Amphibien, Vögeln und Fischen vorkommen. Bestehen auch in den frühen Stadien der regressiven Metamorphose manche Differenzpunkte, so schwinden dieselben gegen Ende der Umwandlungserscheinungen mehr und mehr und machen durchaus gleichen Befunden Platz. - 


\section{Erklärung der Abbildungen.}

Fig. 1. Eierstock von Lacerta agilís, an welchem 2 Eier zum grössten Teil aus ihrem Zusammenhang mit dem Ovarium gelöst wurden. Die Kidechse war 22 Tage nach dem Eingriff getötet. Gefässwucherung auf den operierten Eiern. Vergröss. etwa 2/1. Daneben der Eierstock der gesunden Seite.

Fig. 2. Stück der dorsalen Leibeswand einer Eidechse, an dem noch beide Ovarien hängen. Vergröss. etwa $2^{1 / 4}: 1$. Das eine Ovarium a ist unverändert, an dem anderen $b$ waren einige Eier angestochen. Das Tier wurde 31 Tage nach dem Eingriff getötet. $c$ c sind die in Rückbildung begriffenen, zusammengeklappten Follikel.

Fig. 3. Zwei Ovarien in Zusammenhang mit dem Mesovarium, 23 und 35 Tage nach dem Anstechen der Follikel. Bei a b c d angestochene, zusammengefallene und in Rückbildung begriffene Eier. Vergrössert.

Fig. 4. Schnitt durch ein Ei von den in Fig. 3 wiedergegebenen Ovarien, 23 Tage nach der Operation. Mässige bindegewebige Wandverdickung. Epithelien - zum Teil in verschiedenen Stadien des Zerfalls - das ganze Innere auskleidend. Follikelinhalt ist Eiweissgerinnsel.

Fig. 5. Schnitt durch ein anderes Ei von demselben Ovarium: die Epithelien sind spärlicher geworden, vom vorherrschenden Bindegewebe überwuchert. Leukocyten im Eilumen, das keine vollkommen epitheliale Auskleidung mehr aufweist. Bei Ep. Epithelreste.

Fig. 6. Durchschnittsbild von einem Ei aus späterer Zeit der Umwandlung 31 Tage nach der Operation. Nur noch wenig Epithelien Ep. finden sich. Vorherrschend ist das Bindegewebe, das mit zerfallenden Wanderzellen das Lumen fast ausfüllt.

Fig. 7. Schnitt durch die Mitte eines grossen Follikels ans noch späterer Zeit. Man sieht die zusammengeklappten Wandungen, in denen die Epithelien gänzlich fehlen. Im Lumen Bindegewebssprossen. 
Fig. 8. Schnitt durch ein Ovarium im Endstadium der Metamorphose, 38 Tage p. Op.; a. Kuppe eines gesunden Eies. b. Rest bindegewebiger Natur von einem grösseren umgewandelten Ei. c. Rest eines kleineren rückgebildeten Eies. Bei b. ist die Abgrenzung gegen das Mesovarium als kernreichere Linie zu erkennen.

Figg. 1-3 Reproduktionen von Photogrammen von Prof. Strahl.

Figg. 4-8 sind Zeichnungen nach Präparaten mit Vergrösserung Leitz Oc. I Obj. 3. 